\title{
TRIANGULAR FUZZY-ROUGH SET BASED FUZZIFICATION OF FUZZY RULE-BASED SYSTEMS
}

\author{
Janusz T. Starczewski ${ }^{1, *}$, Piotr Goetzen ${ }^{2}$, Christian Napoli ${ }^{3}$ \\ ${ }^{1}$ Department of Computational Intelligence, Czestochowa University of Technology, \\ al. Armii Krajowej 36, 42-200 Częstochowa, Poland \\ ${ }^{2}$ Information Technology Institute, University of Social Sciences, 90-113 Łódz \\ and Clark University Worcester, MA 01610, USA \\ ${ }^{3}$ Department of Computer, Control and Management Engineering, \\ Sapienza University of Rome \\ Via Ariosto 25 Roma 00185, Italy \\ *E-mail: janusz.starczewski@pcz.pl
}

Submitted: 3rd October 2019; Accepted: 1st May 2020

\begin{abstract}
In real-world approximation problems, precise input data are economically expensive. Therefore, fuzzy methods devoted to uncertain data are in the focus of current research. Consequently, a method based on fuzzy-rough sets for fuzzification of inputs in a rulebased fuzzy system is discussed in this paper. A triangular membership function is applied to describe the nature of imprecision in data. Firstly, triangular fuzzy partitions are introduced to approximate common antecedent fuzzy rule sets. As a consequence of the proposed method, we obtain a structure of a general (non-interval) type-2 fuzzy logic system in which secondary membership functions are cropped triangular. Then, the possibility of applying so-called regular triangular norms is discussed. Finally, an experimental system constructed on precise data, which is then transformed and verified for uncertain data, is provided to demonstrate its basic properties.
\end{abstract}

Keywords: general type-2 fuzzy logic systems, fuzzy-rough fuzzification, regular type-2 t-norms, cropped triangular secondary membership functions

\section{Introduction}

Suboptimal control and erroneous decisions made from inaccurate data are extremely costly. However, precise input data are also economically expensive. Poor data quality in working approximation or control systems may not be a major economic problem if they are trained on precise data. For four decades, automatic control has been the main application area of the fuzzy set theory (e.g. [2]). Although fuzzy controllers operate on fuzzy values, data presented at inputs and data produced at outputs should have accurate values (crisp) as it is the case in the input and output values of controlled systems. Various fuzzification methods are employed to transform the accurate value to the fuzzy values, among which the simplest transformation, called singleton fuzzification, is the most popular. Nevertheless, to model uncertain or noisy data within a fuzzy logic framework, non-singleton fuzzification of inputs is used (eg. [19]). Nonsingleton Fuzzy Logic Systems (FLSs) have shown their capability to make determined efforts to deal with uncertainty. A formal derivation of general 
non-singleton fuzzy logic systems has been provided by Mouzouris and Mendel [15].

Recent works have demonstrated that type- 2 fuzzy sets, i.e. sets characterised by fuzzy subsets of the truth range (see e.g. $[1,22]$ ), are flexible in capturing uncertainty due to their extra degrees of freedom. Also, our previous work [17, 27] has confirmed that type-2 fuzzy logic systems can outperform type-1 FLSs, especially when the fuzzification type of inputs is adequate to uncertainty in applications.

Numerous approaches for identification and modeling nonlinear systems, usually in the presence of uncertainty, have been based on non-singleton fuzzification of interval type-2 fuzzy logic systems IT2FLS [13, 14, 21]. Sahab and Hagras proposed an automatic generation of non-singleton type- 2 fuzzy inputs from data without an assumption of a specific shape about the uncertainty distribution associated with the input [23]. Pekaslan et al. presented a method for dynamic update of interval type-2 fuzzy input sets capturing varying levels of uncertainty affecting systems' inputs [18].

None of the authors has proposed fuzzification of inputs with the help of both the fuzzy and rough set theories combined. This paper relies on the observation that membership functions form triangular fuzzy partitions which approximate antecedent fuzzy sets of rules with the use of the notion of a fuzzy-rough set. To adequately describe the nature of imprecision in data, triangular membership functions are applied in this paper. A triangular membership function is defined by three points: the left boundary, the mode value, and the right boundary. The simplest approach is to estimate these parameters from sample data using the sample maximum and the minimum for the boundaries and making use of any reasonable statistic (e.g. statistical mode, mean, or median) as an estimator for the mode value. Otherwise, expert knowledge can be helpful to estimate the parameters of the triangle, e.g. [5].

As a result, we obtain a structure of a noninterval fuzzy system of type-2, hence it is called a general type-2 fuzzy logic system. The fuzzy-rough method produces quasi-triangular secondary membership functions which are cropped by the limit of any fuzzy truth value. Such memberships can hardly be processed by extended t-norms, evolved from t-norms using the extension principle. We propose to apply so-called regular triangular norms; consequently, their particular forms for cropped triangular membership functions will need to be derived and discussed. To demonstrate the abilities of the whole approach, we construct a fuzzy system of type- 1 and train it on precise data, then transform it into its type-2 version using fuzzy-rough approximation. Verification for poor and uncertain data shows the basic properties of the proposed method.

The paper is organized as follows. Section 2 covers essential elements from the fuzzy set theory and the rough set theory, especially type-2 fuzzy sets, type-2 FLSs and fuzzy-rough sets. Section 3 presents a mathematical bacground for the main idea to use fuzzy-rough sets in triangular fuzzification of triangular system inputs. A new class of secondary membership functions characterizing type- 2 fuzzy sets, further on referred to as cropped triangular membership functions, is introduced in Section 4. Consequently, regular t-norms that operate on cropped membership functions and axiomatic justification for defined t-norms are provided in subsections. Section 5 delivers details for type reduction and final defuzzification of triangular type-2 fuzzy conclusions. Experimental results are summarized in Section 6, while conclusions are drawn in Section 7.

\section{Preliminaries}

In this Section, we describe essential elements from the fuzzy set theory and the rough set theory which are important to the discussion that follows.

\subsection{Type-2 fuzzy sets and systems}

Fuzzy sets of type-2 have been introduced by Zadeh as sets whose membership grades are fuzzy subsets of the unit interval [32].

Definition 1 A type-2 fuzzy subset of a set $\mathbb{X}$ (called also a general type-2 fuzzy set), denoted by $\tilde{A}$, is a vague collection of elements characterized by membership function $\mu_{\tilde{A}}: \mathbb{X} \rightarrow \mathcal{F}([0,1])$, where $\mathcal{F}([0,1])$ is a set of all classical fuzzy sets in the unit interval $[0,1]$.

$\mathbb{X}$ is called a primary domain. A function $f_{x} \in \mathcal{F}([0,1])$ mapping $f_{x}:[0,1] \rightarrow[0,1]$, associ- 
ated with each $x \in \mathbb{X}$, is called a secondary membership function. The fuzzy membership grade $\mu_{\tilde{A}}(x)$ is a fuzzy truth value, since it belongs to the power set of fuzzy subsets of the truth interval $[0,1]$.

Generally, a fuzzy truth value, $F$, is a fuzzy subset of the unit interval $[0,1]$. Only convex fuzzy truth values have linguistic interpretation like somehow high, more or less medium, and definitely low. Therefore, we often restrict $\mathcal{F}([0,1])$ to fuzzy truth numbers which are additionally normal.

Definition 2 A fuzzy truth value $F$ with a membership function $f$ is called a fuzzy truth interval if it is normal at a unique number, i.e. $\exists ! u \in$ $[0,1] \quad f(u)=1$, and convex, i.e. $\forall u_{1}, u_{2}, \lambda \in$ $[0,1], f\left(\lambda u_{1}+(1-\lambda) u_{2}\right) \geqslant \min \left(f\left(u_{1}\right), f\left(u_{2}\right)\right)$.

It is important to emphasize that fuzzy truth numbers are a more general concept than intervals in interval type-2 fuzzy sets. In this paper, we characterize fuzzy truth numbers by triangular membership functions.

The set-theoretic intersection is derived as the minimum transformed by the extension principle. Let $\tilde{A}$ be characterized by its fuzzy membership grades $\mu_{\tilde{A}}(x)$, and $\tilde{B}$ is characterized by $\mu_{\tilde{B}}(x)$, $\forall \mu_{\tilde{A}}(x), \mu_{\tilde{B}}(x) \in \mathcal{F}([0,1]), x \in \mathbb{R}$. The standard intersection $\tilde{A} \cap \tilde{B}$ is defined via its fuzzy membership grades calculated by the extension of the minimum operator, denoted by $\mathrm{min}$, i.e.,

$$
\begin{aligned}
\mu_{\tilde{A} \cap \tilde{B}}(x) & =\widetilde{\min }\left(\mu_{\tilde{A}}(x), \mu_{\tilde{B}}(x)\right), \\
\mu_{\mu_{\tilde{A} \cap \tilde{B}}(x)}(w) & =\sup _{\min (u, v)=w} \min \left(f_{x}(u), g_{x}(v)\right),(2)
\end{aligned}
$$

where $f_{x}$ and $g_{x}$ are bounded membership functions of fuzzy membership grades $\mu_{\tilde{A}}(x)$ and $\mu_{\tilde{B}}(x)$, respectively. More forms of the intersection can be derived on extensions of other t-norms $\widetilde{T}$.

The fundamental structure of a type-2 fuzzy logic system [7] consists of four basic blocks, i.e. the fuzzifier, rule base, inference engine and defuzzifier that have to deal with type-2 fuzzy sets. The rule base is formed by $K$ rules $\widetilde{R}_{k}$ : IF $\widetilde{A}^{\prime}$ is $\widetilde{A}_{k}$ THEN $\widetilde{B}^{\prime}$ is $\widetilde{B}_{k}$, where $\widetilde{A}^{\prime}$ is a type- 2 fuzzified $N$-dimensional input $\mathbf{x}, \widetilde{B}^{\prime}$ is a type- 2 conclusion fuzzy set, $\widetilde{A}_{k}$ is an $N$-dimensional antecedent fuzzy set of type-2, and $\widetilde{B}_{k}$ is a consequent fuzzy set, $k=1, \ldots, K$. The most particular is the type-2 defuzzifier, which usually is decomposed into two sub-blocks: a type reducer and the type-1 (ordinary) defuzzifier.

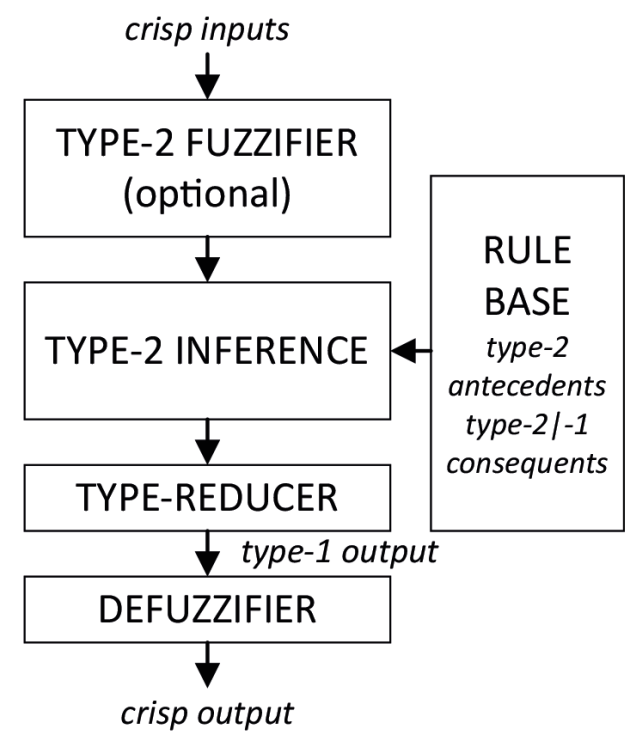

Figure 1. Fuzzy logic system of type-2

The individual rule conclusion, given by the compositional rule of inference $\widetilde{B}^{\prime}{ }_{k}=\widetilde{A}^{\prime} \circ\left(\widetilde{A}_{k} \mapsto\right.$ $\left.\widetilde{B}_{k}\right)$, can be obtained using an extended version of sup- $T$ composition, i.e.

$$
\mu_{\widetilde{B}_{k}^{\prime}}(y)=\sup _{\mathbf{x} \in \mathbf{X}}\left\{\widetilde{T}\left(\mu_{\widetilde{A}^{\prime}}(\mathbf{x}), \widetilde{R}\left(\mu_{\widetilde{A}_{k}}(\mathbf{x}), \mu_{\widetilde{B}_{k}}(y)\right)\right)\right\} .
$$

If input values $\mathbf{x}^{\prime}$ are singleton type-2 fuzzy sets without any special form of fuzzification, relations are realized by conjuctions and consequents are singletons (or taken using the extended height type defuzzification), then composition (3) reduces to the following form

$$
\mu_{\widetilde{B}_{k}^{\prime}}=\mu_{\widetilde{A}_{k}}\left(\mathbf{x}^{\prime}\right)=\widetilde{T}_{n=1}^{N}\left(\mu_{\widetilde{A}_{k, n}}\left(x_{n}^{\prime}\right)\right) .
$$

\subsection{Fuzzy-rough sets}

A rough set is an approximation of an object in a crisp approximation space. This concept has been linked to the concept of a fuzzy set in a general and flexible interpretation of a fuzzy-rough set proposed by Nakamura [16]. An equivalence relation has been extended to a fuzzy relation $R$ on $\mathbb{X}$, which is reflexive $\left(\mu_{R}(x, x)=1 \forall x \in \mathbb{X}\right)$, symmetric $\left(\mu_{R}(x, y)=\mu_{R}(y, x) \forall x, y \in \mathbb{X}\right)$, and transitive $\left(\mu_{R}(x, z) \geq \sup _{y} \min \left(\mu_{R}(x, y), \mu_{R}(y, z)\right) \quad \forall x, y, z \in\right.$ $\mathbb{X})$. Thus, it can be decomposed into $\alpha$-cuts. In the original definition, the fuzzy-rough set is an $\alpha$ composition of rough-fuzzy sets. However, many 
fuzzy equivalence relations form a fuzzy partition on $\mathbb{X}$ by fuzzy sets $F_{i}$. The fuzzy partition $\Phi$ has to cover the whole $\mathbb{X}$, i.e.,

$$
\inf _{x} \max _{i} \mu_{F_{i}}(x)>0 .
$$

Therefore, a fuzzy-rough set may also be interpreted as a pair $\{\underline{\Phi}(A), \bar{\Phi}(A)\}$ of the lower and upper approximations of $A$ by a family of fuzzy partition sets $\left\{F_{i}\right\}$.

Definition 3 Let $\mathbb{X}$ be a set, $X$ be a subset of $\mathbb{X}$ and $F_{i}$ form fuzzy equivalence relations on $\mathbb{X}$. A fuzzyrough approximation of a fuzzy set $A$ is a composition of upper and lower rough approximations of $A$, by $\alpha$-cuts of $F_{i}$, characterized by

$$
\begin{aligned}
& \bar{\Phi}_{i, \alpha}(A)=\sup \left\{\mu_{A}(x) \mid x \in\left[F_{i}\right]_{\alpha}\right\}, \\
& \underline{\Phi}_{i, \alpha}(A)=\inf \left\{\mu_{A}(x) \mid x \in\left[F_{i}\right]_{\alpha}\right\},
\end{aligned}
$$

In [26], we have noticed that the composition of $\alpha$-cuts $\bigcup_{\alpha \in(0,1]}\left[\Phi_{i, \alpha}(A), \bar{\Phi}_{i, \alpha}(A)\right]$ formally represents a fuzzy grade of type-2. Such fuzzy-rough sets are usually defined on weak fuzzy partitions $\Phi$ on $\mathbb{X}$ with disjoint subpartition fuzzy sets $F_{i}$ satisfying $\sup _{x} \min \left(\mu_{F_{i}}(x), \mu_{F_{j}}(x)\right)<1 \forall i \neq j$. The partitions are much stronger if we consider a continuous family of fuzzy partitions, e.g. triangular, which is a key to a particular fuzzification method.

\section{Triangular fuzzy-rough set based fuzzification}

A key approach to non-singleton fuzzification is to represent it by a generalized fuzzifcation function $\mu_{F}\left(x, x^{\prime}\right)=\mu_{F_{i}}(x)$ which is written as an explicit membership function of the mode $x^{\prime}$. This representation allows us to derive all constructions of fuzzyrough sets for a continuous spectrum of $x^{\prime}$ values. The definition of fuzzy-rough sets [16] leads us directly to non-singleton fuzzification, where triangular fuzzy partitions $F_{i}$ reflect the uncertainty of input data around an input value $x^{\prime}$. Assuming the antecedent fuzzy set $A_{k, n}$ and the partition $F_{i}$ are fuzzy numbers, i.e. continuous, single-peak and monotone on slopes, the triangular fuzzy-rough set is ex- pressed as in $[26,27]$ :

$$
\begin{aligned}
& f_{n}\left(u, x_{n}^{\prime}\right) \\
& =\max \left(\mu_{F_{n}}\left(\underline{\mu}_{A_{k, n}}^{-1}(u), x_{n}^{\prime}\right), \mu_{F_{n}}\left(\bar{\mu}_{A_{k, n}}^{-1}(u), x_{n}^{\prime}\right)\right),
\end{aligned}
$$

where $k$ stands for a rule number and $n$ is an input. The equation represents the secondary membership function of the antecedent $A_{k, n}$ after fuzzification. In the case of symmetric and monotone-on-slopes continuous fuzzy truth numbers, the same function can be expressed by cases [26, 27], i.e.

$$
f_{n}\left(u, x_{n}^{\prime}\right)= \begin{cases}\mu_{F_{n}}\left(\bar{\mu}_{A_{k, n}}^{-1}(u), x_{n}^{\prime}\right) & \text { if } m_{k, n} \leq x_{n}^{\prime} \\ \mu_{F_{n}}\left(\underline{\mu}_{A_{k, n}}^{-1}(u), x_{n}^{\prime}\right) & \text { otherwise }\end{cases}
$$

where $m_{k, n}$ is the mode of $A_{k, n}$.

\subsection{Triangular Fuzzification of Triangular MFs}

In our approach, both $A_{k, n}$ and $F_{n}$ are symmetric triangular fuzzy numbers, i.e. $\mu_{A_{k, n}}\left(x_{n}\right)=$ $/ \frac{x_{n}-m_{k, n}+\delta_{k, n}}{\delta_{k, n}}, \frac{m_{k, n}-x_{n}+\delta_{k, n}}{\delta_{k, n}} / \quad$ and $\quad \mu_{F_{n}}\left(x_{n}\right)=$ $/ \frac{x_{n}-x_{n}^{\prime}+\Delta_{n}}{\Delta_{n}}, \frac{x_{n}^{\prime}-x_{n}+\Delta_{n}}{\Delta_{n}} /$, where $m_{k, n}$ and $x_{n}^{\prime}$ denote modes, $\delta_{k, n}$ and $\Delta_{k, n}$ denote spreads of triangular membership functions. A boundary operator introduced as $|z|=\max (0, \min (1, z))$ preserves the output of the functions to be in $[0,1]$. Consequently, the secondary membership function of the fuzzified antecedent can be expressed as follows

$$
f_{k, n}\left(u, x_{n}^{\prime}\right)=\left\{\begin{array}{c}
/ \min \left(\begin{array}{c}
\frac{\delta_{k, n}+m_{k, n}-x_{n}^{\prime}+\Delta_{n}-\delta_{k, n} u}{\Delta_{n}}, \\
\frac{\delta_{k, n} u-\delta_{k, n}+x_{n}^{\prime}-m_{k, n}+\Delta_{n}}{\Delta_{n}}
\end{array}\right) / \\
\quad \text { if } m_{k, n} \leq x_{n}^{\prime} \\
/ \min \left(\begin{array}{c}
\frac{\delta_{k, n} u-\delta_{k, n}+m_{k, n}-x_{n}^{\prime}+\Delta_{n}}{\Delta_{n}}, \\
\frac{\delta_{k, n}+x_{n}^{\prime}-m_{k, n}+\Delta_{n}-\delta_{k, n} u}{\Delta_{n}}
\end{array}\right) / \\
\text { otherwise. }
\end{array}\right.
$$

This function has a triangular shape for $x_{n}^{\prime} \in$ $\left[m_{k, n}-\delta_{k, n}, m_{k, n}+\delta_{k, n}\right]$. However, it is not a general case, for now, let us exclude the case when the result is not triangular. The principal membership function is an original, not fuzzified antecedent function

$$
\widehat{\mu}_{A_{k, n}}\left(x_{n}^{\prime}\right)=\mu_{A_{k, n}}\left(x_{n}^{\prime}\right) .
$$


We search for the support of the secondary membership function, then, we observe that $f_{k, n}\left(u, x^{\prime}\right)=0$ for all $u \in[0,1]$ whenever $m_{k, n}-\delta_{k, n} \geq x_{n}^{\prime}+\Delta_{n}$ or $m_{k, n}+\delta_{k, n} \leq x_{n}^{\prime}-\Delta_{n}$. Therefore, the upper membership function is characterized by the trapezoidal membership function

$$
\bar{\mu}_{A_{k, n}}\left(x_{n}^{\prime}\right)=/ \min \left(\begin{array}{c}
\frac{x_{n}^{\prime}-m_{k, n}+\Delta_{n}+\delta_{k, n}}{\delta_{k, n}} \\
\frac{m_{k, n}+\Delta_{n}-x_{n}^{\prime}+\delta_{k, n}}{\delta_{k, n}}
\end{array}\right) /
$$

having a trivial kernel $\left[m_{k, n}-\Delta_{n}, m_{k, n}+\Delta_{n}\right]$. Consequently, the lower membership function is triangular and supported by $\left[m_{k, n}-\delta_{k, n}+\Delta_{n}, m_{k, n}+\delta_{k, n^{-}}\right.$ $\Delta_{n}$ ] and subnormal. It has a mode at $x_{n}^{\prime}=m_{k, n}$ that can be calculated in the following way

$$
\begin{aligned}
h_{k, n} & =\mu_{A_{k, n}}\left(m_{k, n}+\Delta_{n}\right) \\
& =/ \frac{m_{k, n}-\left(m_{k, n}+\Delta_{n}\right)+\delta_{k, n}}{\delta_{k, n}} / \\
& =/ 1-\frac{\Delta_{n}}{\delta_{k, n}} / .
\end{aligned}
$$

Therefore, the lower membership function is composed of the two following slopes scaled by $h_{k, n}$, i.e.

$$
\begin{aligned}
\underline{\mu}_{A_{k, n}}\left(x_{n}^{\prime}\right)= & h_{k, n} / \min \left(\begin{array}{c}
\frac{x_{n}^{\prime}-m_{k, n}-\Delta_{n}+\delta_{k, n}}{\delta_{k, n}}, \\
\frac{m_{k, n}-\Delta_{n}^{\prime} x_{n}^{\prime}+\delta_{k, n}}{\delta_{k, n}}
\end{array}\right) / \\
= & / 1-\frac{\Delta_{n}}{\delta_{k, n}} / . \\
& / \min \left(\begin{array}{c}
\frac{x_{n}^{\prime}-m_{k, n}-\Delta_{n}+\delta_{k, n}}{\delta_{k, n}} \\
\frac{m_{k, n}-\Delta_{n}-x_{n}^{\prime}+\delta_{k, n}}{\delta_{k, n}}
\end{array}\right) / .
\end{aligned}
$$

The construction of secondary membership functions is demonstrated in Figure 2. Three exemplary $x^{\prime}$ values have served to construct three exemplary secondary membership functions. To obtain a continuous type- 2 fuzzified antecedent set, $\mu_{F}\left(x, x^{\prime}\right)$ should take arguments in the whole spectrum of $x^{\prime}$ values.

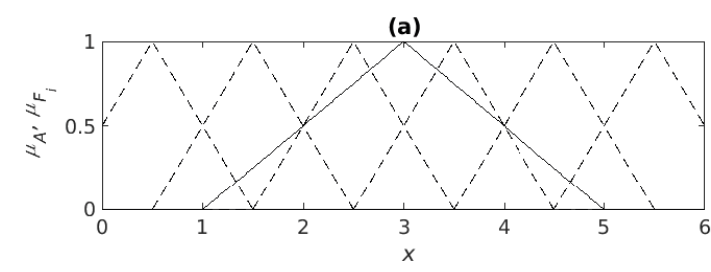

(b)

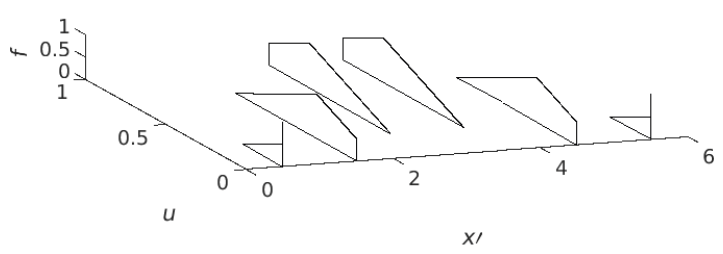

Figure 2. Construction of fuzzy-rough sets: a) $A^{k}$ - antecedent membership function (solid line), $\mu_{F_{i}}$ - placement of non-singleton premise membership functions (dashed lines), b) $f\left(u, x^{\prime}\right)$ - corresponding secondary membership functions.

Unfavorably, for $x_{n}^{\prime} \notin\left[m_{k, n}-\delta_{k, n}, m_{k, n}+\delta_{k, n}\right]$, the intersection between the fuzzy partition set and the antecedent fuzzy set is not sufficient; hence, secondary memberships of the result are no longer triangular. In the sequel, however, when using new operations on type-2 fuzzy sets, we will lose this non-triangularity, as we used triangular approximations for the clipped secondary membership functions.

\section{Cropped Triangular Secondary MFs}

Suppose $x_{n}^{\prime}>m_{k, n}+\delta_{k, n}$ when the secondary membership function of the fuzzy-rough set is no longer triangular. In such a case, the secondary membership function is cropped by the $f$-axis and 10 for $u>0$ becomes

$$
f_{k, n}\left(u, x_{n}^{\prime}\right)=\frac{\delta_{k, n}+m_{k, n}-x_{n}^{\prime}+\Delta_{n}-\delta_{k, n} u}{\Delta_{n}} .
$$

Obviously, the secondary membership function is normal when $u=0$, since $\mu_{A_{k, n}}\left(x_{n}^{\prime}\right)=0$, and therefore it is not continuous. Although the principal membership grade is equal to zero, a line along the slope of the secondary membership function crosses $f$-axis at $\mu_{F_{n}}\left(m_{k, n}+\delta_{k, n}\right)$. The function $f_{k, n}$ 
on an extended $u$-range reaches 1 at some negative point

$$
\widehat{\mu}_{A_{k, n}}\left(x_{n}^{\prime}\right)=\frac{\delta_{k, n}+m_{k, n}-x_{n}^{\prime}}{\delta_{k, n}},
$$

thus it is not a membership grade; however, it allows for the exact calculation of the secondary membership when the fuzzification function $\mu_{F_{n}}$ does not sufficiently overlap the antecedent function $\mu_{A_{k, n}}$. Therefore, we formalize a new type of a quasi-triangular function which includes all possible results of the triangular fuzzy-rough fuzzification method.

Definition 4 Consider ordered real parameters $\lambda \leq \pi \leq v$ with $v \geq 0$, and $v>1$ whenever $\pi<$ 1 , and $\lambda \leq 0$ whenever $\pi \geq 0$ or $\lambda=\pi$. A cropped triangular secondary membership function $\phi(u):[0,1] \rightarrow[0,1]$ such that

$$
\begin{aligned}
\phi(u) & =\operatorname{crptrg}(u, \lambda, \pi, v) \\
& = \begin{cases}\max \left(0, \frac{v-u}{v-\pi}\right) & \text { if } u \geq \pi>0 \\
\max \left(0, \frac{u-\lambda}{\pi-\lambda}\right) & \text { if } u<\pi \\
1 & \text { if } u=0 \text { and } \pi \leq 0 .\end{cases}
\end{aligned}
$$

With regard to semantics, $v$ is an upper membership grade and $\pi$ will be called an improper principal membership since there is no condition for $\pi$ to be greater than zero. Analogically, $\lambda$ will be called an improper lower membership grade.

\section{Theorem 1 The following properties are true:}

- $\phi$ is normal (at a unique point),

- $\phi$ is a convex fuzzy set,

- $\phi$ is left semi-continuous.

Proof - For non-positive $\pi, \phi$ yields exactly 1 for $u=0$. In the case of positive $\pi$, the function is normal at the peak of a triangle.

$-\phi$ is a convex fuzzy set due to the linearity of slopes.

- In the case of positive $\pi$, the function is continuous since the left slope and the right slope yield the same value at $\pi$. In the case of $\pi=0$, the function is also continuous since the limit of the right slope is equal to 1 as $u$ approaches 0 . Otherwise, the function returns 1 at 0 and $\lim _{u \rightarrow 0^{+}} \max \left(0, \frac{v-u}{v-\pi}\right)=\lim _{u \rightarrow 0^{+}} \frac{v-u}{v-\pi}<1$, therefore it is left semi-continuous.

Normality and fuzzy-convexity restrict fuzzy truth values to the class of fuzzy truth numbers, which is required for the implementation of extended operations as type-2 operations [25, 27], such as extended t-norms or t-conorms; however, semi-continuity will complicate this kind of implementation.

\subsection{T-norm approximation}

To extend set-theoretical operations of classical fuzzy set theory, many scientists employ the generalized extension principle. This approach gives a general framework for calculating secondary membership grades, e.g. fuzzy grades of a t-norm operation on fuzzy truth values.

Definition 5 Let $F$ and $G$ be fuzzy truth values, with their membership functions $f$ and $g$, respectively, at $x \in \mathbb{R}$, where for simplicity $x$ is omitted, and let $T$ and $T_{*}$ be arbitrary t-norms. An extended $t$-norm $T$ based on $T_{*}$ according to the generalized extension principle, denoted by $\tilde{T}_{\left\langle T_{*}\right\rangle}(F, G)$, is characterized by the following membership function,

$$
\mu_{\tilde{T}_{\left\langle T_{*}\right\rangle}(F, G)}(w)=\sup _{T(u, v)=w} T_{*}(f(u), g(v)) .
$$

We treat this framework as a basic reference for developing alternative engineering operations to be computationally efficient and convergent to the basic ones. The simplest formulation relies on the use of, so-called, regular operations by calculating only three vertex points of triangular as well as quasitriangular functions. This approach relieves operations computationally and, more importantly, preserves the shape of the result so that it does not exceed the class of input functions, which in this paper are cropped triangular secondary membership functions. In this paper, we extend the scope of regular t-norms [27, 29] to cropped triangular arguments.

An uncomplicated formulation of the regular minimum t-norm of type-2 for cropped triangular membership functions can compute both proper and 
improper membership parameters using the minimum t-norm. Let the input type-2 fuzzy sets be characterized for each $x \in \mathbb{R}$ by fuzzy truth values, whose secondary membership functions are cropped triangular.

Definition 6 Having cropped triangular membership functions $f=\operatorname{crptrg}\left(u, \lambda_{f}, \pi_{f}, v_{f}\right)$ and $g=$ $\operatorname{crptrg}\left(u, \lambda_{g}, \pi_{g}, v_{g}\right)$, a regular minimum t-norm of type-2, denoted by $\widehat{\min }(F, G)$, is characterized by $\operatorname{crptrg}(u, \lambda, \pi, v)$ for each $x \in \mathbb{R}$ with parameters

$$
\begin{aligned}
& v=\min \left(v_{f}, v_{g}\right), \\
& \pi=\min \left(\pi_{f}, \pi_{g}\right), \\
& \lambda=\min \left(\lambda_{f}, \lambda_{g}\right),
\end{aligned}
$$

where the dependence of secondary membership functions on $x$ has been omitted for simplicity reasons.

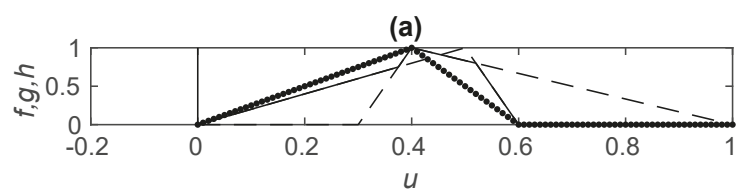

(b)
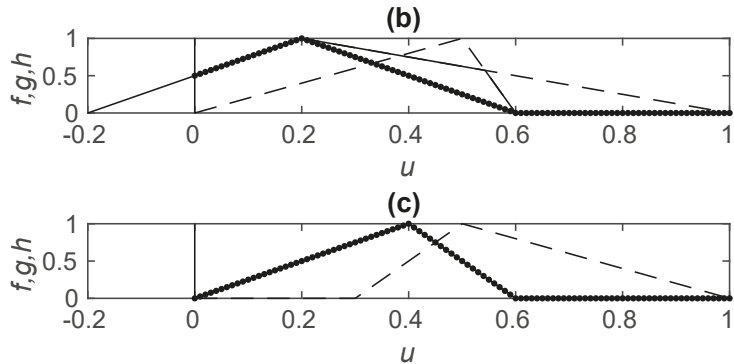

Figure 3. Regular minimum t-norm (dotted) for cropped triangular functions (dashed); extended minimum (solid)

In most cases, there is no difference between the regular minimum t-norm and the exact (minimum-based) extension of the minimum tnorm on cropped triangular membership functions (e.g. Fig.fig:minTcropp c)). Two peculiar cases are shown in Figure 3 a) and b). Using the minimum t-norm, concerning improper membership grades, raises no objections; however, an application of the product t-norm is no longer straightforward. In the case of cropped triangles, only proper membership parameters can be directly calculated with the use of a type-1 t-norm, e.g. the product t-norm can be used for upper membership grades as it can be observed in Fig.fig:prodTcropp c).

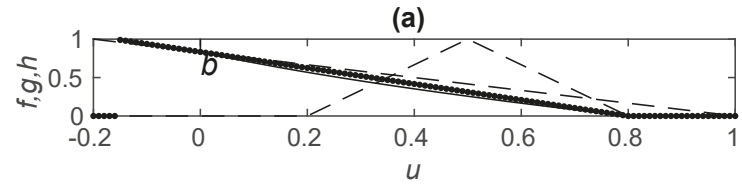

(b)

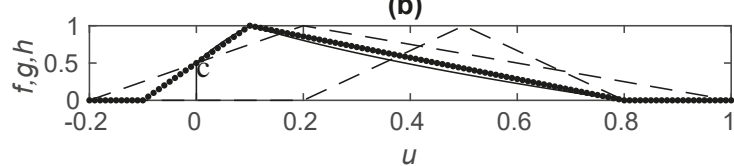

(c)

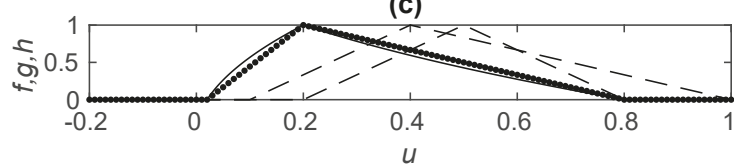

Figure 4. Regular product t-norm (dotted) for cropped triangular functions (dashed); extended product t-norm (solid)

Basically, we have to make an approximating assumption that the operation preserves the cropped triangular shape at the output. Then, we observe that the product can be directly applied only to proper upper membership grades and positive values of principal and lower grades. In the case of at least one negative input value of the improper principal membership grades, the output principal parameter is negative and could be calculated as the argument of the linear function along the upper slope of the resultant secondary membership function reaching value 1 . The function should intersect the primary membership axis at the same point $(0, b)$ as one of the input upper slopes does it as well. The case can be observed in Figure4 a). Suppose there exists at least one of the intersection points of the lines along input upper slopes and the secondary membership axis

$$
\begin{aligned}
b_{f} & =\frac{v_{f}}{v_{f}-\pi_{f}} \text { if } \pi_{f}<0, \\
b_{g} & =\frac{v_{g}}{v_{g}-\pi_{g}} \text { if } \pi_{g}<0 .
\end{aligned}
$$

By the definition of the extended t-norm, the minimal intersection point has to be chosen, i.e.

$$
b=\min \left(b_{f}, b_{g}\right) .
$$

A line along the upper slope of the resultant membership function intersects with the $f$-axis at $b$, i.e.

$$
f=\frac{-b}{v} u+b
$$


The function reaches 1 at the following improper principal grade

$$
\begin{aligned}
f(\pi) & =1 \\
\pi & =v\left(1-\frac{1}{b}\right) .
\end{aligned}
$$

In the case of proper output principal membership grades and at least one negative improper input lower membership grade, the output lower grade is negative and could be calculated as the zero of the linear function along the lower slope of the resultant secondary membership function. The function should intersect the primary membership axis at the same point $(0, c)$ as one (the greates) of the input lower slopes does it as well. This case can be observed in Figure 4 b). Suppose there exists at least one of the intersection points of lines along input lower slopes and the secondary membership axis

$$
\begin{aligned}
c_{f} & =\frac{-\lambda_{f}}{\pi_{f}-\lambda_{f}} \text { if } \pi_{f}<0, \\
c_{g} & =\frac{-\lambda_{g}}{\pi_{g}-\lambda_{g}} \text { if } \pi_{g}<0 .
\end{aligned}
$$

By the definition of the extended t-norm, the maximal intersection point has to be chosen,

$$
c=\max \left(c_{f}, c_{g}\right) .
$$

A line along the lower slope of the resultant membership function intersects with the $f$-axis at $c$ and has the zero at the following improper lower grade:

$$
\lambda=\frac{\pi}{1-\frac{1}{c}} \text {. }
$$

Definition 7 Having cropped triangular membership functions $f=\operatorname{crptrg}\left(u, \lambda_{f}, \pi_{f}, v_{f}\right)$ and $g=$ $\operatorname{crptrg}\left(u, \lambda_{g}, \pi_{g}, v_{g}\right)$, a regular product $t$-norm of type-2, denoted by $\widetilde{\Pi}(F, G)$, is characterized by $\operatorname{crptrg}(u, \lambda, \pi, v)$ for each $x \in \mathbb{R}$ with parameters

$$
\begin{aligned}
& v=v_{f} v_{g} \\
& \begin{cases}\pi_{f} \pi_{g} & \text { if } \pi_{f}>0 \\
& \text { and } \pi_{g}>0\end{cases} \\
& \pi=\left\{\begin{array}{l}
v_{f} v_{g} . \\
\left(1-\frac{1}{\min \left(\frac{v_{f}}{v_{f}-\pi_{f}}, \frac{v_{g}}{v_{g}-\pi_{g}}\right)}\right) \text { otherwise, }
\end{array}\right.
\end{aligned}
$$

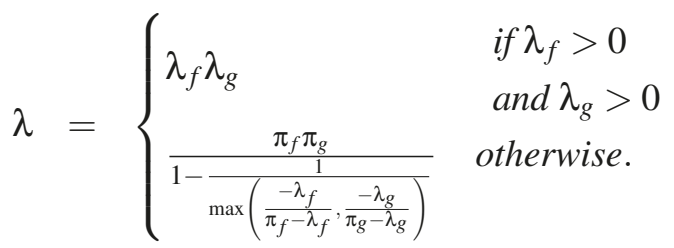

where the dependence of secondary membership functions on $x$ has been omitted for simplicity reasons.

\subsection{Axiomatic justification for t-norms}

Suppose we have two fuzzy truth values $F$ and $G$ characterized by their membership functions $f:[0,1] \rightarrow[0,1]$ and $g:[0,1] \rightarrow[0,1]$. Mizumoto and Tanaka [12] introduced a relation ordering fuzzy truth values using the extended minimum $\widetilde{\min }$ and the extended maximum $\widetilde{\max }$ in the forms of

$$
\begin{aligned}
& \widetilde{\min }(F, G)=\sup _{\min (u, v)=w} \min (f(u), g(v))(39) \\
& \widetilde{\max }(F, G)=\sup _{\max (u, v)=w} \min (f(u), g(v))(40)
\end{aligned}
$$

Thereby, the ordering relation is defined as

$$
\begin{aligned}
& F \sqsubseteq G \Longleftrightarrow \widetilde{\min }(F, G)=F \\
& \text { and } \widetilde{\max }(F, G)=G
\end{aligned}
$$

In the case of fuzzy truth numbers, e.g. cropped triangles, it can be easily verified that the ordering relation defined by both (41) and (42) is equivalent to the following both (not equivalent) inequalities

$$
\begin{array}{ll}
f(w) \geqslant g(w) & \text { if } w \in\left[0, m_{F}\right], \\
f(w) \leqslant g(w) & \text { if } w \in\left[m_{G}, 1\right],
\end{array}
$$

where $m_{F}$ and $m_{G}$ are the peak values of $F$ and $G$, respectively.

Let $\mathcal{L}=(\mathcal{F}([0,1]), \sqsubseteq)$ denote an underlying lattice of the fuzzy set theory, where $\mathcal{F}([0,1])$ is the power set of fuzzy truth values. We indicate the smallest element of $\mathcal{L}$ as $\mathbf{0}=1 / 0$, and the largest element as $\mathbf{1}=1 / 1$. An axiomatic definition of triangular norms on $\mathcal{L}$ can be summarized as follows:

Definition 8 A t-norm on the complete lattice $\mathcal{L}=$ $(\mathcal{F}([0,1]), \sqsubseteq)$ (a type-2 $t$-norm for short) is a function of two variables $\tilde{T}: \mathcal{F}([0,1]) \times \mathcal{F}([0,1]) \rightarrow$ $\mathcal{F}([0,1])$ that satisfies
1. monotonicity: $\tilde{T}(F, H) \sqsubseteq \tilde{T}(G, H) \quad$ if $F \sqsubseteq G$,
2. commutativity: $\tilde{T}(F, G)=\tilde{T}(G, F)$,
3. associativity: $\tilde{T}(\tilde{T}(F, G), H)=\tilde{T}(F, \tilde{T}(G, H))$, 
4. existence of the unit element: $\tilde{T}(F, \mathbf{1})=F$,

while $F, G, H \in \mathcal{F}([0,1])$.

Type-2 t-norms which preserve the triangular shape have been studied in [24]. Let a complete lattice $\mathcal{L}=\left(\mathcal{F}_{c \Delta}([0,1])\right.$, $)$ of cropped triangular fuzzy truth numbers be bounded by the unity element expressed as $\mathbf{1}=\operatorname{crptrg}(u, 1,1,1)$ and the zero element expressed by $\mathbf{0}=\operatorname{crptrg}(u, 0, \pi, v)$. The following theorems substantiate the application of regular t-norms in type-2 fuzzy logic systems.

Theorem 2 The regular minimum t-norm $\widehat{\min }(F, G)$ expressed by $(23-25)$ is a t-norm on $\mathcal{L}=\left(\mathcal{F}_{c \Delta}([0,1]), \sqsubseteq\right)$ (of type- 2 ).

Proof Inclusion of cropped triangular fuzzy truth numbers, denoted by $F \sqsubseteq G$, means that $\lambda_{f} \leq \lambda_{g}$, $\pi_{f} \leq \pi_{g}$ and $v_{f} \leq v_{g}$. For every fuzzy truth number $H, \lambda_{f h}=\min \left(\lambda_{f}, \lambda_{h}\right)$ and $\lambda_{g h}=\min \left(\lambda_{g}, \lambda_{h}\right)$, the inequality $\min \left(\lambda_{f}, \lambda_{h}\right) \leq \min \left(\lambda_{g}, \lambda_{h}\right)$ is satisfied by the property of any non-extended t-norm whenever $\lambda_{f} \leq \lambda_{g}$; similarly $\pi_{f h} \leq \pi_{g h}$ and $v_{f h} \leq v_{g h}$. Therefore, monotonicity is proved.

Commutativity and associativity of the minimum-based type-2 t-norm are satisfied since the minimum t-norm is commutative and associative.

The unit element does not change $F$ since $\pi_{f}=$ $\pi_{f} \cdot 1, v_{f}=v_{f} \cdot 1$ and $\lambda_{f}=\lambda_{f} \cdot 1$.

Theorem 3 The regular product t-norm $\widetilde{\Pi}(F, G)$ expressed by $(36-38)$ is a t-norm on $\mathcal{L}=$ $\left(\mathcal{F}_{c \Delta}([0,1]), \sqsubseteq\right)$ (of type-2).

Proof For every fuzzy truth number $H, v_{f h}=v_{f v h}$ and $v_{g h}=v_{g} v_{h}$, the inequality $v_{f} v_{h} \leq v_{g} v_{h}$ is satisfied by the property of any non-extended t-norm whenever $v_{f} \leq v_{g}$. The inequality $\pi_{f} \pi_{h} \leq \pi_{g} \pi_{h}$ is satisfied by the property of any t-norm whenever $\pi_{f}$ $\leq \pi_{g}$ and $\pi_{f}>0$ and $\pi_{g}>0$. In the cases of at least one negative $\pi_{f}$ or $\pi_{g}$,

$$
\begin{aligned}
& v_{f} v_{h}\left(1-\frac{1}{\min \left(\frac{v_{f}}{v_{f}-\pi_{f}}, \frac{v_{h}}{v_{h}-\pi_{h}}\right)}\right) \\
& \leq v_{g} v_{h}\left(1-\frac{1}{\min \left(\frac{v_{g}}{v_{g}-\pi_{g}}, \frac{v_{h}}{v_{h}-\pi_{h}}\right)}\right)
\end{aligned}
$$

$$
\begin{aligned}
\min \left(\begin{array}{c}
\frac{v_{f}}{v_{f}-\pi_{f}}, \\
\frac{v_{h}}{v_{h}-\pi_{h}}
\end{array}\right) & \leq \min \left(\begin{array}{c}
\frac{v_{g}}{v_{g}-\pi_{g}} \\
\frac{v_{h}}{v_{h}-\pi_{h}}
\end{array}\right) \\
\frac{v_{f}}{v_{f}-\pi_{f}} & \leq \frac{v_{g}}{v_{g}-\pi_{g}} \\
\frac{v_{f}-\pi_{f}}{v_{f}} & \geq \frac{v_{g}-\pi_{g}}{v_{g}} \\
\frac{\pi_{f}}{v_{f}} & \leq \frac{\pi_{g}}{v_{g}}
\end{aligned}
$$

where (46) is valid since $v_{f} \leq v_{g}$ and the component of $H$ is omitted in (47). At least $\pi_{f}$ is negative, $\pi_{f} \leq \pi_{g}$, both sides in the inequality $v_{f} \leq v_{g}$ are positive; therefore, (49) and thus $\pi_{f h} \leq \pi_{g h}$ is valid.

The inequality $\lambda_{f} \lambda_{h} \leq \lambda_{g} \lambda_{h}$ is satisfied by the property of any t-norm whenever $\lambda_{f} \leq \lambda_{g}$ and $\lambda_{f}>$ 0 and $\lambda_{g}>0$. In the cases of at least one negative $\lambda_{f}$ or possibly $\lambda_{g}$, we demonstrate that $v_{f h} \leq v_{g h}$, i.e.

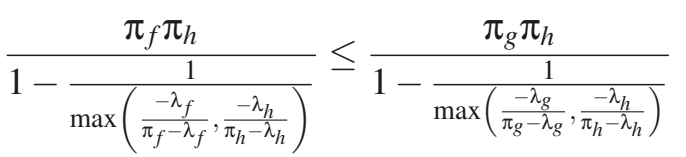

$$
\begin{aligned}
& \max \left(\begin{array}{c}
\frac{-\lambda_{f}}{\pi_{f}-\lambda_{f}}, \\
\frac{-\lambda_{h}}{\pi_{h}-\lambda_{h}}
\end{array}\right) \geq \max \left(\begin{array}{c}
\frac{-\lambda_{g}}{\pi_{g}-\lambda_{g}}, \\
\frac{-\lambda_{h}}{\pi_{h}-\lambda_{h}}
\end{array}\right) \\
& \frac{\lambda_{f}}{\pi_{f}-\lambda_{f}} \leq \frac{\lambda_{g}}{\pi_{g}-\lambda_{g}} \\
& \frac{\pi_{f}-\lambda_{f}}{\lambda_{f}} \geq \frac{\pi_{g}-\lambda_{g}}{\lambda_{g}} \\
& \frac{\lambda_{f}}{\pi_{f}} \leq \frac{\lambda_{g}}{\pi_{g}} .
\end{aligned}
$$

Therefore, monotonicity is proved.

Commutativity and associativity of the productbased type- $2 \mathrm{t}$-norm are satisfied since the product $\mathrm{t}$-norm is commutative and associative.

The unit element does not change $F$ since $\pi_{f}=$ $\pi_{f} \cdot 1, v_{f}=v_{f} \cdot 1$ and $\lambda_{f}=\lambda_{f} \cdot 1$.

Although the analyzed regular t-norms on cropped triangular fuzzy truth numbers represent only approximate extensions of the minimum $t$ norm and the product t-norm, they are still t-norms of type-2, and consequently, their use is justified for the conjunction of type- 2 antecedents as well as for the Cartesian product. 


\section{Type reduction and defuzzifica- tion}

Type reduction is the first phase to defuzzify type-2 fuzzy conclusions, i.e., to transform a type2 fuzzy set into a type- 1 fuzzy set. Exact methods rely on the use of the extension principle applied to the centroid or height type-2 defuzzification method $[6,8,33]$.

A computationally efficient method has been proposed in $[27,28]$. It is fully coincident with the extended centroid that includes the Karnik-Mendel type-reduction algorithm (KM algorithm in its basic [7] or enhanced version saving about $50 \%$ of computations [30]).

Theorem 4 [27] Suppose we have a triangular fuzzy-valued (type-2) fuzzy set of ordered discrete primary values $y_{k}$ with their secondary membership functions specified by the upper, principal and lower membership grades, $\bar{\mu}_{k}>\widehat{\mu}_{k}>\underline{\mu}_{k}, k=$ $1,2, \ldots, K$. Let us assume that the KM algorithm determined an interval centroid fuzzy set $\left[y_{\min }, y_{\max }\right]$ for the interval-valued fuzzy set constituted by the upper and lower membership grades. Moreover, let $y_{\mathrm{pr}}$ be a centroid of the principal membership grades calculated by

$$
y_{\mathrm{pr}}=\sum_{k=1}^{K} \frac{\widehat{\mu}_{k} y_{k}}{\widehat{\mu}_{k}}
$$

Then, the centroid of the triangular type-2 fuzzy set is characterized by the following membership function:

$$
\mu(y)= \begin{cases}\frac{y-y_{\text {left }}(y)}{\left(1-q_{1}(y)\right) y+q_{1}(y) y_{\mathrm{pr}}-y_{\text {left }}(y)} & \text { if } y \in\left[y_{\mathrm{min}}, y_{\mathrm{pr}}\right] \\ \frac{y-y_{\mathrm{right}}(y)}{\left(1-q_{\mathrm{r}}(y)\right) y+q_{\mathrm{r}}(y) y_{\mathrm{pr}}-y_{\mathrm{right}}(y)} & \text { if } y \in\left[y_{\mathrm{pr}}, y_{\mathrm{max}}\right],\end{cases}
$$

where the parameters are expressed by

$$
\begin{aligned}
& q_{1}(y)=\frac{\sum_{k=1}^{K} \widehat{\mu}_{k}}{\sum_{k=1}^{K} \overleftarrow{\mu}_{k}(y)}, \\
& q_{\mathrm{r}}(y)=\frac{\sum_{k=1}^{K} \widehat{\mu}_{k}}{\sum_{k=1}^{K} \vec{\mu}_{k}(y)},
\end{aligned}
$$

and

$$
\begin{aligned}
y_{\text {left }}(y) & =\frac{\sum_{k=1}^{K} \overleftarrow{\mu}_{k}(y) y_{k}}{\sum_{k=1}^{K} \overleftarrow{\mu}_{k}(y)} \\
y_{\text {right }}(y) & =\frac{\sum_{k=1}^{K} \vec{\mu}_{k}(y) y_{k}}{\sum_{k=1}^{K} \vec{\mu}_{k}(y)}
\end{aligned}
$$

with

$$
\begin{aligned}
& \overleftarrow{\mu}_{k}(y)= \begin{cases}\bar{\mu}_{k} & \text { if } y_{k} \leq y \\
\underline{\mu}_{k} & \text { otherwise }\end{cases} \\
& \vec{\mu}_{k}(y)= \begin{cases}\bar{\mu}_{k} & \text { if } y_{k} \geq y \\
\underline{\mu}_{k} & \text { otherwise }\end{cases}
\end{aligned}
$$
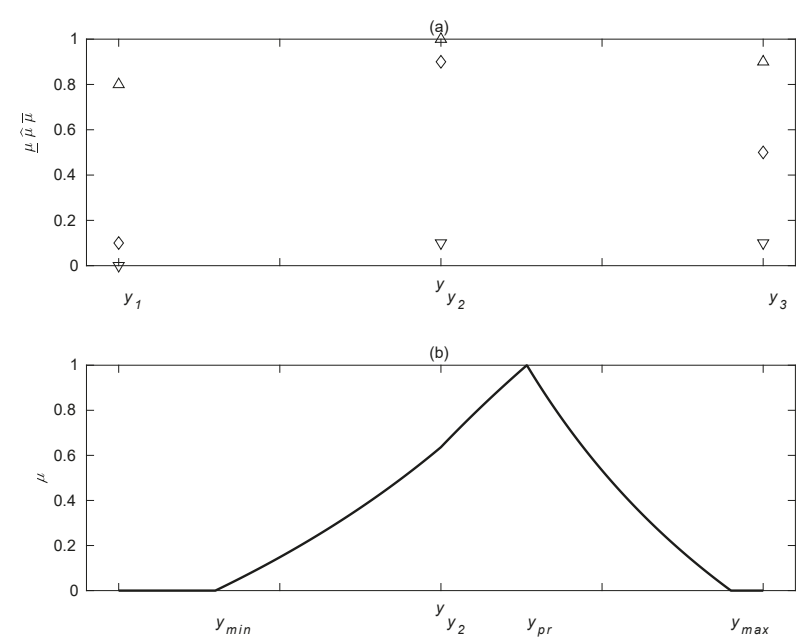

Figure 5. Exemplary quasi-triangular centroid of type-2 fuzzy sets with triangular secondary membership functions: (a) $\triangle-$ upper, $\diamond-$ principal and $\nabla$ - lower membership grades; (b)

$$
\text { centroid fuzzy set }
$$

The second phase of the process is to defuzzify into a crisp value, which can be approximated linearly by:

$$
y^{\prime}=\frac{y_{\text {left }}+y_{\text {pr }}+y_{\text {right }}}{3}
$$

A detailed discussion on a hyperbolic type of approximation which is closer to the exact centroid is presented in [27].

The proposed type-reduction algorithm is actually independent of an interval type-reduction method. Without any modification, we can employ other methods like [10, 31, 4, 3]; however, some of them as the Nie-Tan method [9] or its improvement [11] calculate the centroid directly, without the intermediate type-reduction phase, and are not always good approximations [20].

\section{Experiments}

To conduct experiments showing the essence of fuzzification, we had to train a fuzzy system on non-fuzzified data. In laboratories, we usually have 
available exact data of some approximation or control tasks. Alternatively, we can use an expert approach to construct a base of type-1 fuzzy rules, especially in classification. It is fundamentally important that the thus created system should have maximum accuracy since it is used to initialize a type2 fuzzy logic system, which is more equipped to deal with distorted data. Actually, the initial type-1 fuzzy system becomes the principal component of the type-2 fuzzy system that will not be subject to learning from now on.

Real industrial systems work on uncertain data. Estimating knowledge about the shape of the distortion, we can apply the fuzzy-rough fuzzification method presented in this paper to obtain cropped triangular type-2 fuzzy rules, which completes the transformation of the type-1 fuzzy system into the type-2 fuzzy system. It needs to be noted that this transformation is carried out once ahead-of-time. Consequently, our experiments can be carried out in the following order:

1. As an initial system, the type-1 fuzzy logic system is trained on whole sets of laboratory (exact) data with the use of Back Propagation method (10 folds cross-validation, the system of the highest accuracy is chosen); however, in the cases of unsatisfactory accuracy, more sophisticated methods can be used. The system is constructed on asymmetric-triangular antecedent membership functions (with the singleton fuzzification), rules are fired by the algebraic Cartesian product, and singleton consequents are used in the height type defuzzification. Systems in the problems of the Nonlinear Dynamic Plant approximation and the Kinematics prediction consist of 6 and 13 rules, respectively. The trained system is a reference singleton fuzzy system for further comparisons.

2. As input data are assumed to be distorted by additive noise with a symmetrical triangular distribution, adequate upper and improper principal and lower membership functions are computed for cropped triangular secondary memberships according to the proposed fuzzification method.

3. The data distorted by white additive noise with the triangular distribution and symmetrical spread values $\Delta_{i}$ (for particular $i$-th input) are used for testing of the systems being compared as in the real-time environment. All results are averaged from 10 epochs of generations of distorted samples.

Errors for type-2 fuzzy logic systems obtained via the triangular fuzzy-rough fuzzification of triangular membership functions in the Nonlinear Dynamic Plant approximation are summarized in Tables 1 and 2. The main comparison is made in relation to the type-1 fuzzy logic system. The results are also referenced to a corresponding interval type-2 fuzzy system composed of the upper and lower membership functions of the triangular type2 fuzzy system, which is equivalent to a roughfuzzy approach to fuzzification. It can be observed that the cropped triangular fuzzy system gives lower root mean square error (RMSE) than singleton and interval fuzzy systems at low levels of data distortion.

Table 1. Nonlinear Dynamic Plant approximation with triangular type-2 fuzzy (fuzzy-rough) sets induced by triangular fuzzification of triangular membership functions and additional triangular noise applied to a single input $X_{1}$

\begin{tabular}{cccc}
\hline$\Delta_{1}$ & $\begin{array}{c}\text { Singleton } \\
\text { RMSE }\end{array}$ & $\begin{array}{c}\text { Interval } \\
\text { RMSE }\end{array}$ & $\begin{array}{c}\text { Cropped triangular } \\
\text { RMSE }\end{array}$ \\
\hline 0.1 & 0.813 & 0.812 & 0.811 \\
0.2 & 0.853 & 0.865 & 0.850 \\
0.3 & 0.916 & 0.995 & 0.925 \\
0.4 & 1.022 & 1.191 & 1.061 \\
0.5 & 1.150 & 1.463 & 1.268 \\
0.6 & 1.273 & 1.807 & 1.481 \\
0.7 & 1.344 & 2.203 & 1.723 \\
\hline$\Delta_{2}$ & & & \\
\hline 0.1 & 0.823 & 0.819 & 0.820 \\
0.2 & 0.896 & 0.870 & 0.872 \\
0.3 & 1.008 & 0.952 & 0.951 \\
0.4 & 1.144 & 1.065 & 1.050 \\
0.5 & 1.278 & 1.178 & 1.145 \\
0.6 & 1.477 & 1.349 & 1.277 \\
0.7 & 1.649 & 1.499 & 1.393 \\
\hline
\end{tabular}


Table 2. Nonlinear Dynamic Plant approximation with triangular type-2 fuzzy (fuzzy-rough) sets induced by triangular fuzzification of triangular membership functions and additional triangular noise applied to all inputs

\begin{tabular}{cccc}
\hline$\Delta_{i}$ & $\begin{array}{c}\text { Singleton } \\
\text { RMSE }\end{array}$ & $\begin{array}{c}\text { Interval } \\
\text { RMSE }\end{array}$ & $\begin{array}{c}\text { Cropped triangular } \\
\text { RMSE }\end{array}$ \\
\hline 0.1 & 0.837 & 0.845 & 0.840 \\
0.2 & 0.960 & 0.992 & 0.964 \\
0.3 & 1.107 & 1.238 & 1.134 \\
0.4 & 1.311 & 1.599 & 1.367 \\
0.5 & 1.522 & 1.960 & 1.625 \\
0.6 & 1.733 & 2.405 & 1.903 \\
0.7 & 1.931 & 2.865 & 2.155 \\
\hline
\end{tabular}

Table 3. Kinematics predictions with triangular type-2 fuzzy (fuzzy-rough) sets induced by triangular fuzzification of triangular membership functions and additional triangular noise applied to a single input $X_{1-4}$

\begin{tabular}{cccc}
\hline$\Delta_{1}$ & $\begin{array}{c}\text { Singleton } \\
\text { RMSE }\end{array}$ & $\begin{array}{c}\text { Interval } \\
\text { RMSE }\end{array}$ & $\begin{array}{c}\text { Cropped triangular } \\
\text { RMSE }\end{array}$ \\
\hline 0.1 & 0.097 & 0.097 & 0.097 \\
0.2 & 0.097 & 0.097 & 0.097 \\
0.5 & 0.098 & 0.103 & 0.100 \\
0.7 & 0.100 & 0.115 & 0.103 \\
1.0 & 0.104 & 0.145 & 0.119 \\
\hline$\Delta_{2}$ & & & \\
\hline 0.1 & 0.097 & 0.097 & 0.097 \\
0.2 & 0.097 & 0.098 & 0.097 \\
0.5 & 0.099 & 0.113 & 0.103 \\
0.7 & 0.104 & 0.133 & 0.109 \\
1.0 & 0.129 & 0.168 & 0.131 \\
\hline$\Delta_{3}$ & & & \\
\hline 0.1 & 0.097 & 0.097 & 0.097 \\
0.2 & 0.099 & 0.099 & 0.098 \\
0.5 & 0.106 & 0.110 & 0.106 \\
0.7 & 0.112 & 0.127 & 0.113 \\
1.0 & 0.125 & 0.162 & 0.129 \\
\hline$\Delta_{4}$ & & & \\
\hline 0.1 & 0.097 & 0.097 & 0.097 \\
0.2 & 0.098 & 0.098 & 0.098 \\
0.5 & 0.103 & 0.112 & 0.103 \\
0.7 & 0.109 & 0.126 & 0.111 \\
1.0 & 0.122 & 0.159 & 0.129 \\
\hline & & &
\end{tabular}

Table 4. Kinematics predictions with triangular type-2 fuzzy (fuzzy-rough) sets induced by triangular fuzzification of triangular membership functions and additional triangular noise applied to a single input $X_{5-8}$

\begin{tabular}{cccc}
\hline$\Delta_{5}$ & $\begin{array}{c}\text { Singleton } \\
\text { RMSE }\end{array}$ & $\begin{array}{c}\text { Interval } \\
\text { RMSE }\end{array}$ & $\begin{array}{c}\text { Cropped triangular } \\
\text { RMSE }\end{array}$ \\
\hline 0.1 & 0.097 & 0.097 & 0.097 \\
0.2 & 0.098 & 0.098 & 0.097 \\
0.5 & 0.103 & 0.107 & 0.103 \\
0.7 & 0.107 & 0.119 & 0.108 \\
1.0 & 0.118 & 0.147 & 0.122 \\
\hline$\Delta_{6}$ & & & \\
\hline 0.1 & 0.098 & 0.097 & 0.097 \\
0.2 & 0.099 & 0.099 & 0.098 \\
0.5 & 0.108 & 0.119 & 0.107 \\
0.7 & 0.119 & 0.141 & 0.121 \\
1.0 & 0.143 & 0.178 & 0.147 \\
\hline$\Delta_{7}$ & & & \\
\hline 0.1 & 0.098 & 0.097 & 0.097 \\
0.2 & 0.099 & 0.099 & 0.099 \\
0.5 & 0.107 & 0.116 & 0.106 \\
0.7 & 0.119 & 0.141 & 0.120 \\
1.0 & 0.140 & 0.177 & 0.143 \\
\hline$\Delta_{8}$ & & & \\
\hline 0.1 & 0.097 & 0.098 & 0.097 \\
0.2 & 0.098 & 0.099 & 0.098 \\
0.5 & 0.107 & 0.114 & 0.106 \\
0.7 & 0.115 & 0.130 & 0.114 \\
1.0 & 0.133 & 0.163 & 0.135 \\
\hline
\end{tabular}

Table 5. Kinematics predictions with triangular type-2 fuzzy (fuzzy-rough) sets induced by triangular fuzzification of triangular membership functions and additional triangular noise applied to all inputs

\begin{tabular}{cccc}
\hline \multirow{2}{*}{$\Delta_{i}$} & $\begin{array}{c}\text { Singleton } \\
\text { RMSE }\end{array}$ & $\begin{array}{c}\text { Interval } \\
\text { RMSE }\end{array}$ & $\begin{array}{c}\text { Cropped triangular } \\
\text { RMSE }\end{array}$ \\
\hline 0.1 & 0.099 & 0.109 & 0.100 \\
0.2 & 0.105 & 0.151 & 0.121 \\
0.5 & 0.141 & 0.259 & 0.189 \\
0.7 & 0.180 & 0.264 & 0.203 \\
1.0 & 0.250 & 0.265 & 0.262 \\
\hline
\end{tabular}

Experimental results in the Kinematics approximation are shown in Tables 3-5. For single inputs fuzzified, the cropped triangular type- 2 fuzzy system gives slightly fewer errors than the single- 
ton type-2 fuzzy system; however, its performance is much better in comparison to the interval fuzzy system. Unfortunately, when the disturbance is applied to all inputs, the cropped triangular type- 2 fuzzy system cannot outperform the singleton relevant system.

\subsection{Conclusions}

In this paper, we have presented a method for fuzzification of inputs in the fuzzy logic system. The method is based on the construction of fuzzyrough sets. Triangular fuzzy partitions were introduced to approximate common antecedent fuzzy rule sets. As a result, we obtained a general form of the type-2 fuzzy logic system. We applied the cropped version of the triangular membership function to adequately model distortion of data. Such cropped triangular secondary membership functions required a suitable and preserving shape t-norm operator. Consequently, we proposed regular triangular norms for cropped triangular argument functions. The regular minimum t-norm, as well as the regular product $t$-norm, turned out to be good approximations of extended t-norms and, which is likely to justify it better, they fully satisfy axiomatics for $\mathrm{t}$-norms of type- 2 .

The experimental approach was based on the construction of a type-1 fuzzy logic system using precise data and transforming it into its type- 2 version via the proposed fuzzification method. Such an approach is dedicated to real-world approximation problems, in which precise input data for working systems are economically expensive. We observed that the cropped triangular type-2 fuzzy system has the best performance when the corresponding interval system has a better performance than the singleton system. Otherwise, the performance of the cropped triangular type-2 fuzzy system is not much worse than the results of the singleton fuzzy system. This makes the proposed system highly reliable. When all inputs are distorted and require a triangular fuzzification, the cropped triangular type- 2 fuzzy system no longer outperforms the singleton system, which is consistent with our previous theoretical research on the approximate equivalence of type-2 and type- 1 fuzzy logic systems [27]. If we have poor data available for all system inputs, the quality of the whole approximation process should also be questioned.

\section{Acknowledgments}

The project financed under the program of the Minister of Science and Higher Education under the name "Regional Initiative of Excellence" in the years 2019-2022 project number 020/RID/2018/19, the amount of financing PLN 12,000,000.

\section{References}

[1] Almohammadi, K., Hagras, H., Alghazzawi, D., and Aldabbagh, G. Users-centric adaptive learning system based on interval type-2 fuzzy logic for massively crowded e-learning platforms. Journal of Artificial Intelligence and Soft Computing Research 6, 2 (2016), 81-101.

[2] Ferdaus, M. M., Anavatti, S. G., Garratt, M. A., and Pratam, M. Development of c-means clustering based adaptive fuzzy controller for a flapping wing micro air vehicle. Journal of Artificial Intelligence and Soft Computing Research 9, 2 (2019), 99-109.

[3] Greenfield, S., and Chiclana, F. Accuracy and complexity evaluation of defuzzification strategies for the discretised interval type-2 fuzzy set. International Journal of Approximate Reasoning 54, 8 (Oct 2013), 1013-1033.

[4] Greenfield, S., Chiclana, F., Coupland, S., and John, R. The collapsing method of defuzzification for discretised interval type-2 fuzzy sets. Information Sciences 179, 13 (2009), 2055-2069.

[5] Han, Z.-q., Wang, J.-q., Zhang, H.-y., and Luo, X.x. Group multi-criteria decision making method with triangular type-2 fuzzy numbers. International Journal of Fuzzy Systems 18, 4 (Aug 2016), 673684.

[6] Karnik, N. N., and Mendel, J. M. Centroid of a type-2 fuzzy set. Information Sciences 132 (2001), 195-220.

[7] Karnik, N. N., Mendel, J. M., and Liang, Q. Type-2 fuzzy logic systems. IEEE Transactions on Fuzzy Systems 7, 6 (1999), 643-658.

[8] Liu, F. An efficient centroid type-reduction strategy for general type-2 fuzzy logic system. Information Sciences 178, 9 (2008), 2224-2236.

[9] Maowen Nie, and Woei Wan Tan. Towards an efficient type-reduction method for interval type2 fuzzy logic systems. In 2008 IEEE International Conference on Fuzzy Systems (IEEE World Congress on Computational Intelligence) (2008), pp. 1425-1432. 
[10] Melgarejo, M. A fast recursive method to compute the generalized centroid of an interval type-2 fuzzy set. In Proc. NAFIPS 2007 (2007), pp. 190-194.

[11] Mendel, J. M., and Liu, X. Simplified interval type2 fuzzy logic systems. IEEE Transactions on Fuzzy Systems 21, 6 (2013), 1056-1069.

[12] Mizumoto, M., and Tanaka, K. Some properties of fuzzy sets of type-2. Information and Control 31 (1976), 312-340.

[13] MonirVaghefi, H., Rafiee Sandgani, M., and Aliyari Shoorehdeli, M. Interval type-2 adaptive network-based fuzzy inference system (anfis) with type-2 non-singleton fuzzification. In 2013 13th Iranian Conference on Fuzzy Systems (IFSC) (2013), pp. 1-6.

[14] Monirvaghefi, H., and Shoorehdeli, M. A. Modelbased fault detection of a nonlinear system using interval type- 2 fuzzy systems with non-singleton type-2 fuzzification. In The 3rd International Conference on Control, Instrumentation, and Automation (2013), pp. 231-236.

[15] Mouzouris, G. C., and Mendel, J. M. Nonsingleton fuzzy logic systems: theory and application. IEEE Transactions on Fuzzy Systems 5, 1 (1997), 56-71.

[16] Nakamura, A. Fuzzy rough sets. Note on MultipleValued Logic in Japan 9, 8 (1988), 1-8.

[17] Nowicki, R. K., and Starczewski, J. T. A new method for classification of imprecise data using fuzzy rough fuzzification. Information Sciences 414 (2017), 33-52.

[18] Pekaslan, D., Wagner, C., and Garibaldi, J. M. Leveraging it2 input fuzzy sets in non-singleton fuzzy logic systems to dynamically adapt to varying uncertainty levels. In 2019 IEEE International Conference on Fuzzy Systems (FUZZIEEE) (2019), pp. 1-7.

[19] Pourabdollah, A., John, R., and Garibaldi, J. M. A new dynamic approach for non-singleton fuzzification in noisy time-series prediction. In 2017 IEEE International Conference on Fuzzy Systems (FUZZ-IEEE) (2017), pp. 1-6.

[20] Rojas, J. D., Salazar, O., and Serrano, H. Nie-Tan Method and its Improved Version: A Counterexample. IngenierÃa 21 (08 2016), 138 - 153.

[21] Ruiz, G., Pomares, H., Rojas, I., and Hagras, H. The non-singleton fuzzification operation for general forms of interval type-2 fuzzy logic systems. In 2017 IEEE International Conference on Fuzzy Systems (FUZZ-IEEE) (2017), pp. 1-6.
[22] Sadiqbatcha, S., Jafarzadeh, S., and Ampatzidis, Y. Particle swarm optimization for solving a class of type-1 and type-2 fuzzy nonlinear equations. Journal of Artificial Intelligence and Soft Computing Research 8, 2 (2018), 103-110.

[23] Sahab, N., and Hagras, H. An adaptive type-2 input based nonsingleton type-2 fuzzy logic system for real world applications. In 2011 IEEE International Conference on Fuzzy Systems (FUZZ-IEEE 2011) (2011), pp. 509-516.

[24] Starczewski, J. T. Efficient triangular type-2 fuzzy logic systems. International Journal of Approximate Reasoning 50 (2009), 799-811.

[25] Starczewski, J. T. Extended triangular norms. Information Sciences 179 (2009), 742-757.

[26] Starczewski, J. T. General type-2 FLS with uncertainty generated by fuzzy rough sets. In Proc. IEEE-FUZZ 2010 (Barcelona, 2010), pp. 17901795.

[27] Starczewski, J. T. Advanced Concepts in Fuzzy Logic and Systems with Membership Uncertainty, vol. 284 of Studies in Fuzziness and Soft Computing. Springer, 2013.

[28] Starczewski, J. T. Centroid of triangular and Gaussian type-2 fuzzy sets. Information Sciences 280 (2014), 289-306.

[29] Starczewski, J. T., Nowicki, R. K., and Nieszporek, K. Fuzzy-rough fuzzification in general FL classifiers. In Proceedings of the 11th International Joint Conference on Computational Intelligence, IJCCI 2019, Vienna, Austria, September 17-19, 2019 (2019), J. J. M. Guervós, J. Garibaldi, A. Linares-Barranco, K. Madani, and K. Warwick, Eds., ScitePress, pp. 335-342.

[30] Wu, D., and Mendel, J. M. Enhanced karnikmendel algorithms. IEEE Transactions on Fuzzy Systems 17, 4 (2009), 923-934.

[31] Wu, D., and Tan, W. Computationally efficient type-reduction strategies for a type-2 fuzzy logic controller. In Proc. IEEE Fuzzy Conference (Reno, NV, 2005), pp. 353-358.

[32] Zadeh, L. A. The concept of a linguistic variable and its application to approximate reasoning - I. Information Sciences 8 (1975), 199-249.

[33] Zhai, D., and Mendel, J. M. Centroid of a general type-2 fuzzy set computed by means of the centroid flow algorithm. In Proc. IEEE-FUZZ 2010 (Barcelona, 2010), pp. 1-8. 


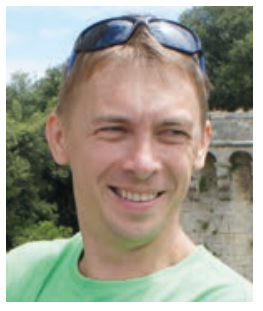

Janusz T. Starczewski is an Associate Professor at Czestochowa University of Technology, where he is Deputy Head of the Department of Intelligent Computer Systems. He holds the Ph.D and D.Sc. degrees in Computer Science and an M.Sc. in Electrical Engineering. He is an experienced project contributor in artificial intelligence and IT systems. The mainstream of his scientific achievements comprise studies on advanced concepts of fuzzy logic, including type-2 fuzzy logic systems and their combinations with the rough set theory. He has authored more than 50 publications. His book "Advanced Concepts in Fuzzy Logic and Systems with Membership Uncertainty" has been granted by the Polish Minister of Science and Higher Education.

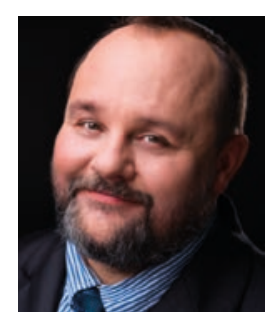

Piotr Goetzen received the Ph.D. in computer chemistry from Université de Neuchâtel, Switzerland. Since his graduation he has been interested in computer science, especially computer networks, operating systems and security of IT systems. Dr. Goetzen leads the Department of Computer Networks at University of Social Sciences.
Highly certified (CCNA, CCNP, CCDA, CCDP, ITIL, Microsoft, Linux), Dr Goetzen has been the IT trainer for more than 20 years. He also works in a security department of one of the global IT Corporations. He is pursuing the research of security of IT systems. He has also been involved in several international projects. Active Erasmus teacher.

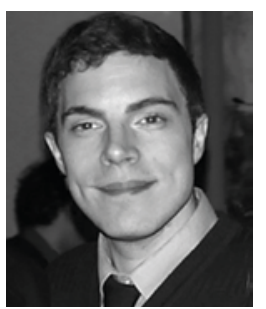

Christian Napoli is an Associate Professor with the Department of Computer, Control, and Management Engineering "Antonio Ruberti”, Sapienza University of Rome, since 2019, as well as Scientific Director and cofounder (2015) of the International School of Advanced and Applied Computing (ISAAC). He received the B.Sc. degree in Physics in 2010, the M.Sc. degree in Astrophysics in 2012 and the Ph.D. in Computer Science in 2016 all from the University of Catania, the National Scientific Abilitation as associate professor in Computer Engineering (2017) and computer science (2019). He is involved in several international research projects, serves as reviewer and member of the board program committee for major international journals and international conferences. His current research interests include neural networks, artificial intelligence, computational models, and high performance computing. 